Enferm Bras 2020;19(4Supl):S26-S33

https://doi.org/10.33233/eb.v19i4.4331

\title{
REVISÃO \\ Atuação da enfermagem no cuidado às pessoas em hemodiálise frente à pandemia do vírus SARS-CoV-2
}

Naiane Pereira de Oliveira*, Eda Schwartz, D.Sc. ${ }^{* *}$, Lilian Moura de Lima Spagnolo, D.Sc. ${ }^{* \star *}$, Tuany Nunes da Cunha ${ }^{\star \star \star *}$, Josiele de Lima Neves, M.Sc. ${ }^{* \star \star \star}$, Fernanda Lise, D.Sc.

*Graduanda em enfermagem pela UFPEL, Pelotas/RS, Bolsista de Iniciação Científica PBIP-AF UFPEL, ${ }^{* \star}$ Enfermeira, Docente da Faculdade de Enfermagem (FEn) e do Programa de PósGraduação da Faculdade de Enfermagem e do Programa de Mestrado Profissional em Saúde da Família da UFPEL, Pesquisadora do NUCCRIN, Bolsista CNPq. Pelotas/RS, ${ }^{* * * E n f e r m e i r a, ~}$ Doutora em Enfermagem. Professora da Faculdade de Enfermagem e do Programa de PósGraduação da Faculdade de Enfermagem da UFPEL, Pelotas/RS, ${ }^{* * * \star E n f e r m e i r a, ~ M e s t r a n d a ~ e m ~}$ Enfermagem pelo Programa de Pós Graduação da UFPE, Pelotas/RS, Bolsista CAPES,

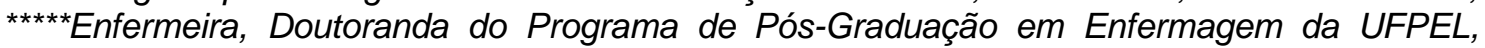

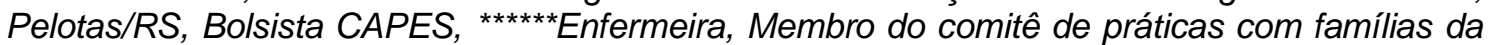
International Family Nursing Association (IFNA) e do Núcleo de Condições Crônicas e suas Interfaces (NUCCRIN) da Faculdade de Enfermagem da UFPel

Recebido em 11 de agosto de 2020; aceito em 18 de agosto de 2020.

Correspondência: Naiane Pereira de Oliveira, Rua Marcílio Dias, 255/203, bloco 10, 96020-480 Pelotas RS

Naiane Pereira de Oliveira: nah3m@hotmail.com

Eda Schwartz: edaschwa@gmail.com

Lilian Moura de Lima Spagnolo: lima.lilian@gmail.com

Tuany Nunes da Cunha: tuanynunes@hotmail.com

Josiele de Lima Neves: josiele_neves@hotmail.com

Fernanda Lise: fernandalise@gmail.com

\section{Resumo}

Objetivo: Conhecer as recomendações para guiar a atuação da Enfermagem às pessoas com doença renal crônica na prevenção e manejos de casos suspeitos e confirmados de COVID-19. Métodos: Trata-se de uma revisão narrativa da literatura, em que foram consultados os websites de organizações não governamentais internacionais para levantar, sintetizar e analisar as diretrizes propostas pelas entidades de nefrologia na abordagem às pessoas em hemodiálise. Resultados: A partir da síntese das recomendações de cinco organizações percebe-se a preocupação dos serviços de hemodiálise em fornecerem informações esclarecedoras e atualizadas aos usuários quanto à prevenção do contágio, ao manejo adequado para os casos suspeitos, e aos cuidados de Enfermagem as pessoas acometidas pelo coronavírus. Conclusão: Essas diretivas poderão ser utilizadas no planejamento dos cuidados pela Enfermagem nos serviços de diálise durante a pandemia.

Palavras-chave: insuficiência renal crônica, diálise renal, cuidados de enfermagem, infecções por coronavírus, pandemias.

\footnotetext{
Abstract

Nursing performance in hemodialysis patient care in the face of the SARS-CoV-2 virus pandemic

Objective: To know the recommendations for guiding the performance of Nursing to people with chronic kidney disease in the prevention and management of suspected and confirmed cases of COVID-19. Methods: This is a narrative review of the literature, in which the websites of international non-governmental organizations were consulted to survey, synthesize and analyze the guidelines proposed by the nephrology entities when approaching people on hemodialysis. Results: Based on the synthesis of the recommendations of five organizations, the hemodialysis services are concerned with providing enlightening and updated information to users regarding
} 
the prevention of contagion, the proper handling of suspected cases, and the nursing care of those affected by the coronavirus. Conclusion: These guidelines can be used in nursing care planning in dialysis services during the pandemic.

Keywords: chronic renal insufficiency, renal dialysis, nursing care, coronavirus infections, pandemics.

\section{Resumen \\ Desempeño de enfermería en la atención de pacientes en hemodiálisis frente a la pandemia del virus SARS-CoV-2}

Objetivo: Conocer las recomendaciones para guiar el desempeño de la Enfermería a las personas con enfermedad renal crónica en la prevención y manejo de casos sospechosos y confirmados de COVID-19. Métodos: Esta es una revisión narrativa de la literatura, en la que se consultó a los sitios web de organizaciones internacionales no gubernamentales para encuestar, sintetizar y analizar las pautas propuestas por las entidades de nefrología al acercarse a las personas en hemodiálisis. Resultados: Con base en la síntesis de las recomendaciones de cinco organizaciones, los servicios de hemodiálisis se preocupan por proporcionar información clara y actualizada a los usuarios sobre la prevención del contagio, el manejo adecuado de casos sospechosos y la atención de enfermería para los afectados por la enfermedad coronavirus. Conclusión: Estas pautas se pueden utilizar en la planificación del cuidado de enfermería en los servicios de diálisis durante la pandemia.

Palabras-clave: insuficiencia renal crónica, diálisis renal, atención de enfermería, infecciones por coronavirus, pandemias.

\section{Introdução}

A COVID-19 foi anunciada pelas autoridades chinesas quando, após pesquisas, identificaram a ocorrência de casos de pneumonia, de origem desconhecida em Wuhan, província de Hubei, em 2019. Assim, associaram a um novo tipo de coronavírus, mais tarde nomeado de SARS-Cov-2 [1]. Em janeiro de 2020 foram relatados os primeiros casos da doença fora da China, rastreados na Tailândia, Japão e República da Coréia, respectivamente. Visto o aumento dos casos no continente asiático, a Organização Mundial da Saúde (OMS) convocou um fórum de pesquisa e inovação, a nível global, para o estudo de um controle da doença, que, pelo alto fator de transmissão e severidade, é declarada em março, como uma pandemia, por estar presente em todos os continentes [2-4].

A nível mundial, no dia 07 de agosto de 2020 haviam 18.902 .735 casos confirmados de COVID-19, com 709.511 óbitos [5]. No Brasil, até a mesma data, haviam 2.912.212 casos confirmados, com 42.720 óbitos relacionados à doença [6] e no que diz respeito aos profissionais de enfermagem estavam reportados 32.648 casos de infecção e 334 óbitos [7].

A doença se manifesta de forma respiratória, sendo transmitida por contato direto com indivíduos e objetos contaminados, ou por via respiratória com a inalação de gotículas emitidas durante a fala, tosse ou espirros. Nesse contexto, foram estabelecidas medidas de prevenção e controle, como o distanciamento social, etiqueta respiratória e o uso das precauções padrão e específicas [8]. Sabe-se que idosos com mais de 60 anos e pessoas com doenças crônicas como Diabetes Mellitus (DM), Hipertensão Arterial Sistêmica (HAS), Doença Renal Crônica (DRC), integram o grupo de risco [9], sendo necessário que medidas de prevenção sejam empregadas pelos pacientes e em ambientes considerados essenciais, como é o caso da unidade de hemodiálise.

A DRC afeta a homeostase corporal e altera principalmente os metabolismos hídricos, eletrolíticos, ácido base e de macronutrientes, levando a propensão de reações pró-inflamatórias, pró-oxidativas e hipercatabólicas [10]. Tais distúrbios criam um processo inflamatório em cadeia, que auxiliam na desnutrição energético-protéica, inibição do apetite e surgimento de anemia. Ainda, prejudicam o sistema cardiovascular e o sistema ósseo com distúrbios no metabolismo mineral e calcificações em vasos e artérias sistêmicas; exacerbação do estresse oxidativo e; alterações do sistema músculo esquelético, causando miopatias urêmicas e influenciando negativamente o sistema respiratório, diminuindo a força e elasticidade de músculos como diafragma e intercostais [11-15].

No Brasil, a Enfermagem apresenta papel essencial no serviço de hemodiálise, uma vez que atua no planejamento e execução de cuidados individualizados, elencados com base nas necessidades do paciente, os quais influenciam na adesão e na qualidade do tratamento [16]. 
Assim, justifica-se a importância deste estudo, tendo em vista a vulnerabilidade do paciente com DRC e que o coloca em real possibilidade de agravamento do caso diante a infecção pela COVID-19. Assim, a atuação da enfermagem na abordagem preventiva e no manejo seguro após o contágio deve ser amparada em diretrizes específicas.

Nesse contexto, o presente estudo objetivou conhecer as recomendações para guiar a atuação da Enfermagem na abordagem às pessoas com doença renal crônica na prevenção, manejo de casos suspeitos e confirmados da COVID-19.

\section{Métodologia}

Trata-se de uma revisão narrativa da literatura, desenvolvida a partir da necessidade de reunir diretivas imediatas, para uma síntese das orientações e conhecimento da Enfermagem sobre a abordagem aos pacientes nos serviços de hemodiálise [17]. Tal metodologia foi utilizada em virtude do conhecimento ora disponível acerca da temática envolvendo a pandemia do vírus SARS-CoV-2. O desenvolvimento envolveu quatro etapas: primeiro, a contextualização da experiência; segundo, a busca por literatura relevante; terceiro, a análise das fontes documentais finais; e quarto, a síntese dos resultados [17].

A contextualização da experiência está relacionada à necessidade de conhecer as recomendações internacionais relacionadas à atuação da Enfermagem, baseada em evidências científicas, no desenvolvimento de estratégias de cuidados das pessoas com DRC em serviços de hemodiálise, decorrente do estado de pandemia pelo vírus SARS-CoV-2.

\section{Coleta dos dados}

Os dados foram coletados por dois revisores, a partir da consulta em websites de entidades não governamentais, nacionais e internacionais, que reúnem especialistas em Nefrologia em nível global, selecionados pelo reconhecimento internacional na apresentação de diretrizes na atenção à saúde das pessoas com DRC. Os cinco websites das entidades consultadas foram: a Sociedade Brasileira de Nefrologia (Brasil); Kidney Care (Reino Unido); National Kidney Fundation (Estados Unidos da América); Leading European Nephrology (Reino Unido) e International Society of Nephrology (Estados Unidos da América e Bélgica).

As buscas foram realizadas entre março e abril de 2020, utilizando materiais disponíveis pelos órgãos desde janeiro de 2020, proporcionais ao início da pandemia. Ressalta-se que foi dada prioridade às atualizações concomitantes as novas descobertas sobre a doença, visto sua singularidade e contemporaneidade.

Análise dos dados

As recomendações foram coletadas em formulário próprio para identificação e organização. A partir da leitura, os dados foram sintetizados para apresentação das diretrizes das fontes para serem utilizadas pela Enfermagem na abordagem as pessoas com DRC em hemodiálise.

Resultados

A síntese das recomendações de cinco organizações internacionais sobre a abordagem aos pacientes em serviços de hemodiálise na prevenção ao contágio, manejo dos casos suspeitos e confirmados de COVID19, foram abordadas sob dois ângulos, sendo, as recomendações para o serviço nas unidades e as recomendações para as abordagens aos pacientes.

Por se tratar de um serviço essencial, a Sociedade Brasileira de Nefrologia elaborou um documento com orientações que devem ser seguidas pelas Unidades de Diálise, sintetizadas no quadro 1 [18].

Por ser um tema atual e de conhecimento escasso, a elaboração das recomendações pela Sociedade Brasileira de Nefrologia leva em consideração as recomendações dos órgãos mundiais de saúde, bem como, as especificações e legislações para o funcionamento de tais unidades no Brasil. Além disso, as adoções destas recomendações devem se adequar ao fluxo e espaço físico do serviço, como a presença de quartos privativos e disposição de dialisadores, deixando a cargo dos coordenadores a melhor forma de implementação. 
No quadro 2, foram apresentadas as recomendações das organizações internacionais para a abordagem à saúde das pessoas com DRC, em tratamento hemodialítico, a fim de evitar a contaminação e manter a continuidade do tratamento, em casos de infecção pela COVID-19 [19-24].

Quadro 1 - Recomendações às unidades de diálise na abordagem dos usuários

Atuar na in formaçăo adequada sobre as medidas básicas de prevençäo e higiene;

Estimular a vacinação dos pacientes, principalmente contra In fluenza;

Desencorajar a presenca de acompanhantes, aqueles que apresentem sintomas respiratórios:

Facilitar avaliação médica de pacientes sintomáticos de in feç̧ão de COVD-19 antes da entrada no local de diálise;

Quando houver quarto privativo para isolamento, pacientes com suspeita ou confirmação de COVD-19 devem ser dialisados em quarto de isolamento;

Quando não houver quarto para isolamento, o(s) pacientes com suspeita ou confirmação de COVD-19 devem dialisar, de preferência, em ala separada e no último tumo;

Em caso de muitos casos suspeitos ou confirmados, recomenda-se, se possivel, tumo de diálise exclusivo ou a abertura de turnos suplementares;

Sugere-se considerar uso único do dialisador em pacientes com casos confirmados ou suspeitos de COVD19;

Manter o paciente sempre com máscaras;

Em pacientes com DRC, suspeitos ou confirmados de COVD-19, não se recomenda a redução do tempo ou frequência do tratamento dialítico.

Quadro 2 - Diretivas para a abordagem aos pacientes em diálise.

\begin{tabular}{|c|c|}
\hline $\begin{array}{l}\text { Organização } \\
\text { Sociedade Brasileira de } \\
\text { Nefrologia }\end{array}$ & $\begin{array}{l}\text { Recomendações e cuidados } \\
\text { Fornecer prescrição extra das medicações de uso crônico regular, } \\
\text { Orientar para evitar viagens não essenciais e aglomeraçôes; } \\
\text { Atentar para sinais de alerta e de emergência como febre e dispneia; } \\
\text { Notificar o serviço de terapia renal substitutiva de referência sobre sintomas de } \\
\text { COVD-19 em pacientes em diálise e discutir a condição de saúde; } \\
\text { Manter isolamento domiciliar por } 14 \text { dias; } \\
\text { Pacientes sintomáticos não devem faltar as sessões de hemodiálise, para isso, a } \\
\text { unidade deve criar um fluxograma ou esquema para evitar o contato destes } \\
\text { pacientes com os demais. }\end{array}$ \\
\hline Kidney Care & $\begin{array}{l}\text { Em caso de sintomas como febre acima de } 37,8^{\circ} \text { ou tosse contínua, o paciente } \\
\text { deve se autoisolar e ficar em casa por sete dias, no caso de morar sozinho. Se } \\
\text { outras pessoas residirem no mesmo local, é necessário isolamento por } 14 \text { dias; } \\
\text { Orientar seguir medidas de distanciamento social; } \\
\text { Pacientes em diálise continuam com as sessóes, podendo ser solicitada em } \\
\text { horários específicos; } \\
\text { Pacientes com DRC devem evitar estritamente o contato com alguém que esteja } \\
\text { apresentando sintomas de coronavíns (COVD-19); } \\
\text { Todos devem utilizar máscaras faciais durante as sessões de diálise. }\end{array}$ \\
\hline $\begin{array}{l}\text { National Kidney } \\
\text { Fundation }\end{array}$ & $\begin{array}{l}\text { Orientar sobre a importância das precauçôes diárias como lavagem das mãos, } \\
\text { distanciamento social, evitar tocar o rosto e cobrir a boca e o nariz ao espirrar; } \\
\text { Se o paciente estiver em diálise, seguir com o tratamento; } \\
\text { Entrar em contato com a dínica de diálise se apresentar algum sintoma } \\
\text { relacionado à COVD-19; } \\
\text { No caso de transplantado, continuar com a medicação imunosupressora, } \\
\text { seguindo as recomendaços da sua equipe de saúde; } \\
\text { Utilizar máscara se precisar sair de casa. }\end{array}$ \\
\hline $\begin{array}{l}\text { Leading European } \\
\text { Nephrology }\end{array}$ & $\begin{array}{l}\text { Recomenda-se aos pacientes ficar em casa nos dias de não diálise e usar } \\
\text { transporte individual para as unidades de diálise; } \\
\text { Usar medidas de etiqueta respiratória e de prevenção do contágio do novo } \\
\text { coronavírus; } \\
\text { Manter seus tratamentos; } \\
\text { Entrar em contato com a dínica de diálise se estiver doente, tiver contato com } \\
\text { individuos com COVD-19 ou se tiver outras preocupações; } \\
\text { Lavar as mãos e o braço da fistula antes de iniciar a diálise e realizar a } \\
\text { antissepsia completa das áreas da punção. } \\
\text { Informar a equipe sobre sintomas respiratórios ou febre, imediatamente após a } \\
\text { chegada ao centro de diálise. }\end{array}$ \\
\hline $\begin{array}{l}\text { International Society of } \\
\text { Nephrology }\end{array}$ & $\begin{array}{l}\text { Orientar sobre a importância de higienizar as mãos frequentemente com água e } \\
\text { sabão; } \\
\text { Evitar sair de casa se estiver doente, exceto para assistência médica; } \\
\text { Manter distanciamento social; } \\
\text { O uso de máscara facial deve ser universal, usadas sempre ao sair de casa. } \\
\text { Evitar contato com pessoas doentes; } \\
\text { Todos os pacientes devem continuar com os medicamentos e doses prescritas. }\end{array}$ \\
\hline
\end{tabular}


Em observação dos aspectos comuns, todas as organizações mantêm o consenso da necessidade de adoção de medidas de distanciamento social, etiquetas de higiene e precauções respiratórias, como o uso universal de máscaras e a continuação de tratamentos e medicamentos essenciais para a manutenção da vida.

A Sociedade Brasileira de Nefrologia aconselha os pacientes sintomáticos a não faltarem as sessões, devendo a unidade de hemodiálise criar um fluxograma para evitar o contato com os demais pacientes, considerando as condições de saúde. Já a National Kidney Fundation e a Leading European Nephrology indicam, para estes pacientes e os que tiveram contato com indivíduos contaminados, o contato prévio com o local de diálise para informações sobre manejos ou possíveis dúvidas.

Para a Sociedade Brasileira de Nefrologia e Leading European Nephology, os pacientes devem-se atentar para febre e sinais de desconforto respiratório, como a dispnéia, mantendo 0 isolamento domiciliar durante 14 dias, para a Kidney Care, após apresentar sintomas de febre acima de $37,8^{\circ}$ ou tosse contínua o paciente deve-se autoisolar por sete dias caso resida sozinho ou 14 dias, se compartilhar a residência com outras pessoas.

Ademais a Leading European Nephrology salienta a necessidade de lavagem de mãos com água e sabão e a importância de realizar a antissepsia no local da fístula e toda região proximal, antes da realização de procedimentos como a punção.

Discussão

Diante das recomendações nacionais e internacionais, evidenciam-se a necessidade dos profissionais oferecerem informações atualizadas sobre o vírus, capazes de apoiar a promoção de cuidados seguros aos pacientes em hemodiálise. Para tanto, é necessário conhecer a etiologia do vírus para, a partir das informações disponíveis, planejarem os cuidados.

Sabe-se que o vírus responsável pela Síndrome Respiratória Aguda Grave (SARS-CoV2) apresenta um alto poder patogênico, infectando o trato respiratório inferior, causando distúrbios pulmonares, associado a um alto índice de letalidade [25]. Estudo realizado por uma Universidade de Hong Kong identificou a taxa de transmissão de 83,0\% em ambiente familiar [26]. No dia a dia, a contaminação pode ocorrer pelo ato de levar a mão à boca, olhos e nariz após entrar em contato com o vírus por apertos de mão, toque em maçanetas e ou outros objetos contaminados ou pelo contato com gotículas salivares, espirro, tosse ou secreções [27], o que impõem a necessidade de medidas preventivas.

Dentro do planejamento da assistência, é fundamental o profissional, com um pensamento crítico e conhecimentos prévios, criar um fluxograma de atendimento, tanto para pacientes que não apresentam nenhuma sintomatologia do COVID-19, como para os pacientes suspeitos e diagnosticados com a doença, visando a diminuição do contágio e proteção à vida de todos. Todas as medidas tomadas devem respeitar as características das unidades de hemodiálise, normas de padronização, capacidade de atendimento, número de profissionais, características socioculturais do ambiente em que está inserida, assim como o perfil dos pacientes e ter referências das recomendações dos órgãos de competências, como Ministérios de Saúde e Sociedades de Nefrologia [18,23,24].

As recomendações preventivas incluem atenção às manifestações da doença. Os principais sintomas da doença são classificados como leve a moderado e incluem tosse, coriza, dor de garganta, febre e diarreia, porém, em pessoas com condições pré-existentes pode levar a depressão do sistema respiratório, causando pneumonia ou insuficiência respiratória aguda com potencial letal [28]. As medidas preventivas para evitar o contágio e controlar infecções correspondem ao uso de máscaras, medidas de etiqueta respiratória, distanciamento social, diminuir o número de acompanhantes nos serviços, lavagem de mãos com água e sabão ou 0 uso de álcool em gel 70\% [18-24].

Destaca-se que as pessoas em tratamento renal, em sua grande maioria, realizam a hemodiálise em três sessões semanais. Havendo, no Brasil, a necessidade de deslocamento até estes serviços com o uso de transporte intermunicipal, muitas vezes coletivo, fornecido pelas Secretarias Municipais de Saúde, em razão de a localidade ser em grandes centros urbanos. Este fato aumenta a exposição e eleva o risco de contágio, visto que há impossibilidade de abandonar o tratamento, indispensável para a manutenção de vida. Diante disso, cabe à Enfermagem fornecer orientações preventivas mediante as recomendações de órgãos competentes, quanto às medidas de higienização e proteção [18-24].

Quanto ao manejo de casos suspeitos, o enfermeiro deve informar sobre os principais sinais e sintomas da COVID-19, orientar sobre qual o procedimento deve ser tomado caso 0 
usuário os apresente; reforçar a importância do comparecimento às sessões de tratamento; auxiliar na elaboração de estratégias para utilização de medicações de uso regular e; reforçar a importância da manutenção do esquema vacinal em dia, especialmente, contra a influenza [1824]. A equipe de enfermagem pode ter uma atuação diferencial neste papel de educador em saúde, pois permanece com o paciente por todo o período em que está conectado a máquina e, em geral, possui elevada credibilidade e confiança do paciente. Em relação aos casos confirmados, adotar caso disponível o uso de único dialisador para este paciente, em sala de isolamento ou em horário diferenciado dos demais [18].

A pessoa com DRC, na maioria das vezes, precisa de auxílio do familiar devido à fragilidade de sua saúde, portanto, tais cuidadores precisam considerar o cuidado com sua saúde, principalmente, para aqueles que realizarem atividades laborais fora da residência. É fundamental que o familiar se sinta acolhido pelo serviço de saúde, pois também acumulam tensões e preocupações pelo caráter excepcional do período da pandemia. Cabe a enfermagem orientá-los a, se possível, quando se ausentarem do serviço evitar aglomerações; fornecer também as informações sobre higienização e prevenção tanto no âmbito domiciliar quanto fora dele, para evitar a exposição do paciente ao vírus e; orientá-los quanto ao fluxograma que devem percorrer para garantia de seus direitos [23,24].

A Enfermagem enquanto profissão que atua na linha de frente no combate da COVID19, combinando o conhecimento científico e o domínio de técnicas para proporcionar cuidado ao paciente, enfrenta diversas dificuldades em seus campos de trabalho, que vão desde a exacerbação da exaustão física, até a predisposição ao aparecimento de condições referentes ao adoecimento mental, pois muitas vezes precisam conciliar mais de um emprego devido ao baixo salário [29].

No setor de hemodiálise, durante o período da pandemia, cabe ao enfermeiro assegurar, o bom funcionamento da unidade, protegendo tanto a equipe de trabalhadores, quanto a saúde dos pacientes. Para oferecer um atendimento de qualidade, deve-se investir em capacitações para a equipe, frisando a importância do trabalho na linha de frente do combate ao vírus e os desafios que serão encontrados [18-24]. Durante uma pandemia, um cenário novo, várias dificuldades serão impostas a estes profissionais, que mesmo com o aumento da exposição a riscos biológicos, o prolongamento do tempo de uso de equipamentos de proteção e a adição de itens para a proteção, terão que encontrar maneiras para acolher com eficácia todos os pacientes e suas demandas.

É inegável a importância destes profissionais para o funcionamento dos sistemas de saúde de todo o mundo, ressaltados no atual contexto, portanto, a sua proteção deve ser garantida, visando à melhoria dos aspectos assistenciais, seja pela articulação das funcionalidades multiprofissionais ou assegurar a qualidade do atendimento e a segurança do paciente.

Vale reforçar que essas orientações têm caráter complementar às diretrizes apresentadas neste estudo, visando o apoio das práticas essenciais da Enfermagem em Nefrologia, durante o período excepcional, acarretado pela pandemia do vírus SARS-CoV-2.

Conclusão

As recomendações das organizações internacionais, não governamentais, poderão ser utilizadas no planejamento seguro dos cuidados pela Enfermagem na abordagem aos pacientes durante a pandemia, nos serviços de Nefrologia. As diretrizes apontam para a necessidade de uso de informações atualizadas e evidências científicas para apoiar as abordagens dos profissionais de saúde, na prevenção da contaminação pelo SARS-CoV-2, na equipe e pacientes, assim como, o manejo dos casos suspeitos e confirmados.

A possível limitação deste estudo pode estar relacionada às características da doença ainda estarem em fase de estudo pelo meio científico, assim como o manejo dos infectados, que podem sofrer alterações, mas que não diminuem a qualidade da informação apresentada neste estudo.

Para a Enfermagem em Nefrologia, o estudo traz recomendações para que seja possível a prática do cuidado seguro durante a pandemia, possibilitando medidas para prevenir a infecção pelo vírus e dar continuidade no tratamento da pessoa com DRC, visando tanto o seu bem-estar e a sua saúde quanto do seu familiar e acompanhante, e a proteção dos membros da equipe, amparados física e mentalmente, que estarão mais do que nunca vulneráveis aos riscos incidentes da profissão. 
1. Pan American Health Organization. COVID 19 situation reports. [Internet] 2020 [citado 2020 abr 28]. Disponível em: https://www.paho.org/en/tag/COVID-19-situation-reports

2. World Health Organization. Emergencies. Diseases. Coronavirus disease 2019. Situation Reports. Situation report - 1. 2020 [citado 2020 maio 13]. Disponível em: https://www.who.int/docs/default-source/coronaviruse/situation-reports/20200121-sitrep1-2019-ncov.pdf?sfvrsn=20a99c10 4

3. World Health Organization. Emergencies. Diseases. Coronavirus disease 2019. Situation Reports. Situation report - 13. 2020 [citado maio 13]. Disponível em: https://www.who.int/docs/default-source/coronaviruse/situation-reports/20200202-sitrep13-ncov-v3.pdf?sfvrsn=195 40106

4. World Health Organization. Emergencies. Diseases. Coronavirus disease 2019. Situation Reports. Situation report - 51. 2020 [citado 2020 maio 13]. Disponível em: https://www.who.int/docs/default-source/coronaviruse/situation-reports/20200311-sitrep51-COVID-19.pdf?sfvrsn=1ba62e57 10

5. World Health Organization. Emergencies. Diseases. Coronavirus disease 2019. Situation Reports. Situation report - 145. 2020 [citado 2020 jun 13]. Disponível em: https://www.who.int/docs/default-source/coronaviruse/situation-reports/20200613COVID-19-sitrep-145.pdf?sfvrsn=bb7c1dc9 2

6. Ministério da Saúde (BR). Painel coronavírus. Brasília DF: 2020 [citado 2020 jun 13]. Disponível em: https://COVID.saude.gov.br/

7. Conselho Federal de Enfermagem (BR). Observatório da enfermagem. Brasília (DF): COFEM 2020 [cited 2020 jun 13]. Disponível em:

http://observatoriodaenfermagem.cofen.gov.br/

8. Pan American Health Organization. Coronavirus infections. [Internet] 2020 [citado 2020 abr 28]. Disponível em: https://www.paho.org/en/topics/coronavirus-infections

9. World Health Organization. Emergencies. Diseases. Coronavirus disease 2019. Advice for public. ASK Who. [Internet] 2020 [citado 2020 mai 28]. Disponível em: https://www.who.int/emergencies/diseases/novel-coronavirus-2019/advice-for-public

10. Baiocco GG. Doença renal crônica: Fisiopatologia e fatores de risco. In: Schwartz; Lise; Satos. Enfermagem em Nefrologia: Interfaces do cuidado na doença renal crônica. $1^{\underline{a}}$ ed. Porto Alegre: Moriá; 2018. p.17-29.

11. Barreto FC, Stinghen AEM, Oliveira RB, Franco ATB, Moreno NA, Barreto DV, et al. Em busca de uma melhor compreensão da doença renal crônica: uma atualização em toxinas urêmica. Braz J Nephrol 2014;36(2):221-23. https://doi.org/10.5935/01012800.20140033

12. Thomé F, Gonçalves LF, Manfro RC, Barros E. Doença renal crônica. In: Barros E, Manfro RC, Thomé FS, Gonçalves LF (eds). Nefrologia: rotinas, diagnóstico e tratamento.3ㄹ. Ed. Porto Alegre: Artes Médicas Sul; 2006: p.381-404.

13. Avesani CM, Carrero JJ, Axelsson J, Qureshi AR, Lindholm B, Stenvinkel P. Inflammation and wastingin chronic kidney disease: Partners in crime. Kidney International 2006;70:S8-S1. https://doi.org/10.1038/sj.ki.5001969

14. Bucharles SGE, Varela AM, Barberato SAH, Pecoits-Filho R. Avaliação e manejo da doença cardiovascular em pacientes com doença renal crônica. Braz J Nephrol 2010;32(1):120-7. https://doi.org/10.1590/S0101-28002010000100019

15. Cury JL, Brunetto AF, Aydos RD. Efeitos negativos da insuficiência renal crônica sobre a função pulmonar e a capacidade funcional. Rev Bras Fisioter 2010;14(2):91-8. https://doi.org/10.1590/S1413-35552010005000008

16. Freitas RLS, Mendonça AEO. Cuidados de enfermagem ao paciente renal crônico em hemodiálise. Carpe Diem: Rev Cult Cient UNIFACEX. 2016;14(2):22-35. Disponível em: https://periodicos.unifacex.com.br/Revista/article/view/678

17. Educação, Grupo Anima. Manual revisão bibliográfica sistemática integrativa: a pesquisa baseada em evidências. [Internet]. 2014 [citado 2020 maio 20]. Disponível em: http://biblioteca.cofen.gov.br/wpcontent/uploads/2019/06/manual revisao bibliografica-sistematica-integrativa.pdf 
18. Sociedade Brasileira de Nefrologia. Recomendações de Boas Práticas da Sociedade Brasileira de Nefrologia às Unidades de Diálise em relação à epidemia do novo Coronavírus (COVID-19). São Paulo SP: SBN; 2020.

19. Kidney Care UK. Coronavirus (COVID-19) guidance for patients with kidney disease. [Internet] 2020 [citado 2020 abr 29]. Disponível em: https://www.kidneycareuk.org/news-and-campaigns/coronavirus-advice/

20. National Kidney Fundation. Kidney disease\& COVID-19. 2020 [citado 2020 abr 29]. Disponível em: https://www.kidney.org/coronavirus/kidney-disease-COVID-19

21. European Renal Association (ERA-EDTA). COVID-19 News and Information. [Internet] 2020 [citado 2020 abr 29]. Disponível em: https://www.era-edta.org/en/COVID-19news-and-information/\#toggle-id-2

22. International Society of Nephrology. Recommendations for the Novel Coronavirus 2019 Epidemic 2020 [citado 2020 abr 29]. Disponível em: https://www.theisn.org/COVID19/recommendations

23. Sociedade Brasileira de Nefrologia. Informações para pacientes com Doença Renal Crônica (DRC) sobre a infecção pelo COVID-19 (Coronavírus). São Paulo (SP): SBN; 2020.

24. Sociedade Brasileira de Nefrologia. Recomendações para pacientes pediátricos em hemodiálise, diálise peritoneal e transplantados renais. São Paulo: Sociedade Brasileira de Nefrologia; 2020.

25. Zaki AM, van Boheemen SV, Bestebroer TM, Osterhaus AD, Fouchier RA. Isolation of a novel coronavirus from a man with pneumonia in Saudi Arabia. N Engl J Med 2012;367(19):1814-20. https://doi.org/10.1056/NEJMoa1211721

26. Chan JFW, Yuam S, Kok KH, ToKKw, Chu J, Yang J et al. A familial cluster of pneumonia associated with the 2019 novel coronavirus indicanting person-to-person transmission: a study of a Family cluster. Lancet 2020;395(10223):514-23. https://doi.org/10.1016/S0140-6736(20)30154-9

27. Ministério da Saúde (BR). COVID 19. 2020 [citado 2020 abr 28]. Disponível em: https://coronavirus.saude.gov.br/sobre-a-doenca\#o-que-e-COVID

28. Bai Y, Yao L, Wei T, Tian F, Jin DY, Chen $L$ et al. Presumed asymptomatic carrier transmission of COVID-19. JAMA 2020;323(14):1406-7. https://doi.org/10.1001/jama.2020.2565

29. Oliveira AC. Desafios da Enfermagem frente ao enfrentamento da pandemia da COVID-19. REME Rev Min Enferm 2020;24:e-1302. https://doi.org/10.5935/1415$\underline{2762.20200032}$ 